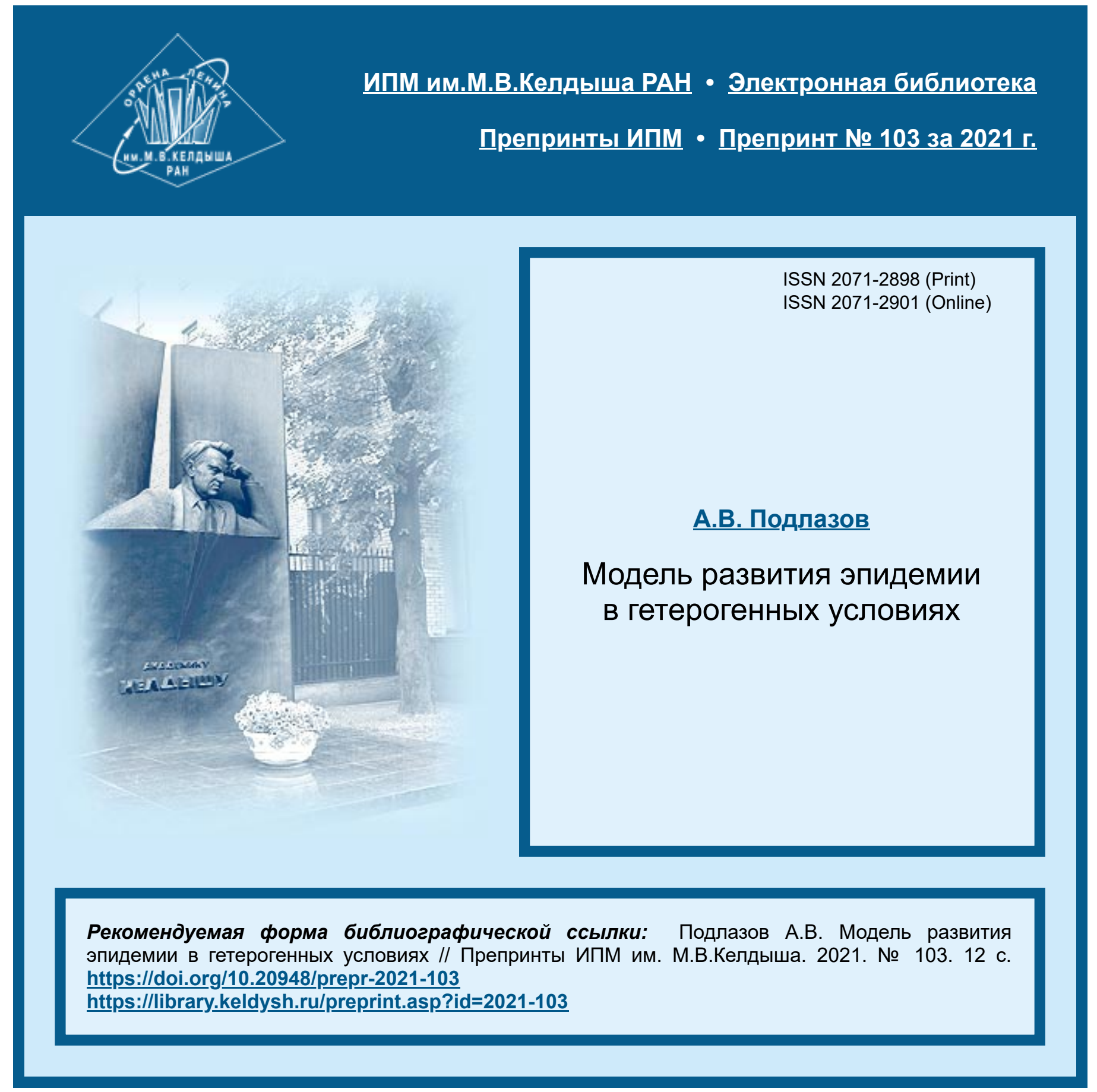




\author{
Орд ен а Л е ни на \\ ИНСТИТУТ ПРИКЛАДНОЙ МАТЕМАТИКИ \\ имени М.В.Келдыша \\ Российской академии наук
}

\author{
А.В. Подлазов \\ Модель развития эпидемии \\ в гетерогенных условиях
}




\section{А.В. Подлазов}

\section{Модель развития эпидемии в гетерогенных условиях}

Предлагаются две модификации SIR-модели распространения эпидемии, учитывающие социальную и пространственную неоднородность популяции. Социальная неоднородность, связанная с различиями в интенсивности парных контактов между людьми, качественно меняет базовое репродуктивное число. Пространственная неоднородность, связанная с различиями в интенсивности множественных контактов между людьми, существенно смещает положение равновесия, увеличивает характерные времена и приводит к возникновению колебательной динамики конечной продолжительности.

Ключевые слова: эпидемиология, статусные модели, SIR-модель, базовое репродуктивное число, суперраспространители, волны эпидемии, коллективный иммунитет

\section{A.V. Podlazov}

\section{Epidemic spreading model in heterogeneous conditions}

I propose two modifications of the SIR-model of the epidemic spread, taking into account the social and space heterogeneity of the population. Social heterogeneity associated with differences in the intensity of paired contacts between people qualitatively changes the basic reproductive number. Space heterogeneity associated with differences in the intensity of multiple contacts between people significantly shifts the equilibrium position, increases the characteristic times and leads to the emergence of oscillatory dynamics of finite duration.

Key words: epidemiology, compartmental models, SIR-model, basic reproduction number, super-spreaders, epidemic waves, herb immunity

Работа выполнена при поддержке РФФИ (проект 19-01-00602).

\section{Содержание}

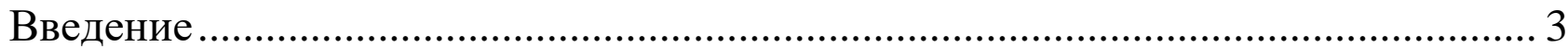

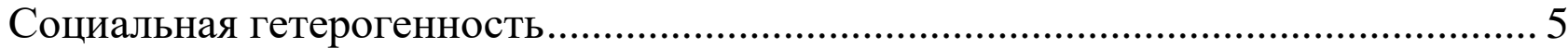

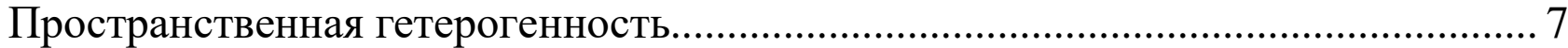

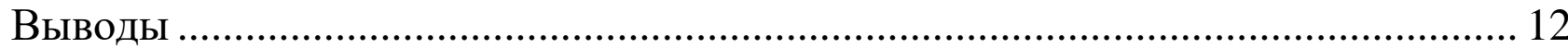




\section{ВВЕДЕНИЕ}

Простейшей моделью развития эпидемии является классическая SIR-модель, названная по переменным $S, I$ и $R$, которыми она оперирует. Они характеризуют доли в популяции людей различного cmamyca (compartment): восприимчивых (Susceptible), инфицированных (Infectious) и переболевших/невосприимчивых (Recovered/Resistant). Последний может трактоваться и более широко - как выбывшие (Removed), т.е. умершие или такие больные, которые уже выявлены и изолированы от здоровых, - в любом случае эти люди утрачивают возможность повторно заражаться сами и заражать восприимчивых. Однако обычно данная модель применяется для описания распространения тех заболеваний, после которых возникает устойчивый иммунитет.

Модель описывает кинетику переходов людей между разными статусами в предположении, что заражение восприимчивых людей происходит в результате их контактов с инфицированными, которые, в свою очередь, постепенно выздоравливают. Если константы скорости этих процессов неизменны, а условия гомогенны, то получается элементарная система из трех уравнений

$$
\left\{\begin{array}{l}
\dot{S}=-r S I \\
\dot{I}=r S I-I, \\
\dot{R}=I
\end{array}\right.
$$

из которых в силу нормировки $S+I+R=1$ независимы лишь два.

Модель имеет единственный параметр $r$ - базовое репродуктивное число. Оно характеризует для полностью восприимчивой популяции число новых заражений, приходящихся на каждого инфицированного в течение периода его контагиозности, который принят за единицу измерения времени.

Состояние $I=0$ является положением равновесия модели, устойчивостью которого определяется реакция популяции на заражение. Величина $I$ будет увеличиваться, пока $r S>1$. Таким образом, если $r>1$, то доля инфицированных может нарастать, пока некоторая часть популяции не переболеет, став невосприимчивой, а если $r<1$, то эпидемия не развивается даже в полностью восприимчивой популяции с $S=1$, что далее будет рассматриваться в качестве начальных условий.

Избыточность модели позволяет взглянуть на развитие эпидемии поиному, используя не второе уравнение системы, а первое и третье. Исключив из них время, получаем автономное уравнение $d S / S=-r d R$, имеющее решение $S=e^{-r R}$. Положения равновесия возникают как решения уравнения $1-R=e^{-r R}$, описывающего состояние популяции без инфицированных людей. Если $r<1$, то оно имеет лишь тривиальное решение $R=0$, а если $r>1$, то обретает еще и нетривиальное решение $0<R^{*}<1$, описывающее итог эпидемии (см. пример на рис. 1), называемый состоянием коллективного иммунитета. 


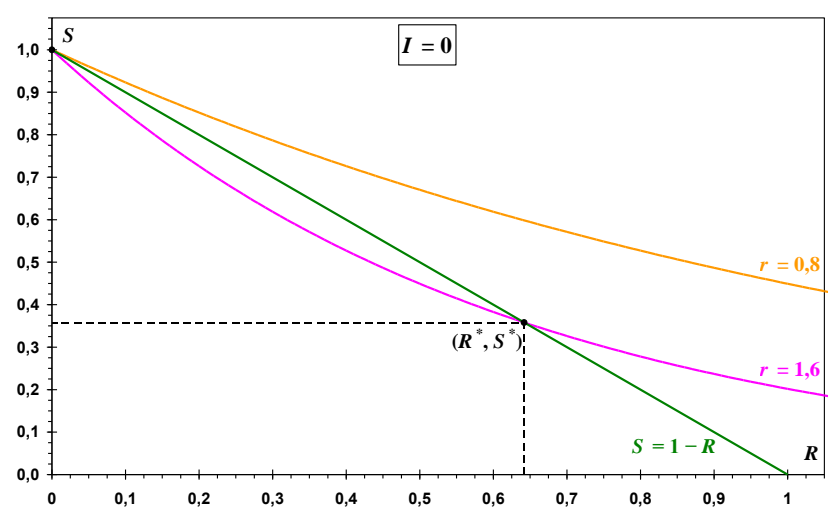

Рис. 1. Положения равновесия

Наличие нетривиальных положений равновесия SIR-модели определяется производной функции $S=e^{-r R}$ в точке $R=0$. При $r<1$ эта функция убывает медленно, так что ее график более не пересекается с прямой $S=1-R$, а при $r>1$ - быстро, чем и обеспечивается дополнительное пересечение.

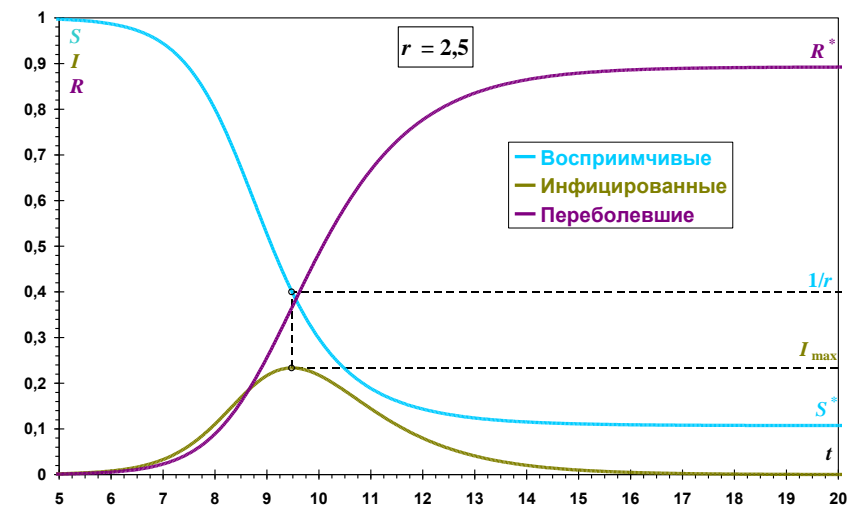

Рис. 2. Пример развития эпидемии

Распространение инфекции замедляется по мере выбытия восприимчивых людей.

Максимум доли инфицированных соответствует именно той доле восприимчивых, которая, имейся она в момент заражения, сделала бы эпидемию невозможной.

Начальные условия: $I(0)=10^{-6}, R(0)=0$.

В предельных случаях можно оценить долю невосприимчивых, необходимых для обретения коллективного иммунитета: $R^{*} \approx 2(r-1)$ для $r-1<<1$ и $R^{*} \approx 1-e^{-r}$ для $r>>1$. При этом $S^{*} \approx 3-2 r$ и $S^{*} \approx e^{-r}$ соответственно.

На рис. 2 представлен типовой пример эволюции доли людей различного статуса. Развитие эпидемии на начальной стадии является экспоненциальным. Пока еще все восприимчивы, то, как следует из уравнения $d I / d R=r S-1$, доля инфицированных растет по закону $I \sim e^{\beta t}$ с $\beta=r-1$. При $S=1 / r$ она достигает пика, высота которого $I_{\max }=1-(1+\ln r) / r$ находится из решения $I=1-S+1 / r \cdot \ln S$. При приближении коллективного иммунитета доля инфицированных спадает по формуле $I \sim e^{-\gamma t}$, где $\gamma=1-r S^{*}$. Анализ показывает, что при $r=1$ отношение коэффициентов $\gamma / \beta=1$, а далее оно монотонно убывает по мере роста $r$, т.е. чем выше базовое репродуктивное число, тем медленнее затухает эпидемия по сравнению со скоростью ее развития.

Если допустить, что переболевшие люди приобретают лишь нестойкий иммунитет, то SIR-модель превращается в SIRS-модель с уравнениями

$$
\left\{\begin{array}{l}
\dot{S}=-r S I+b R \\
\dot{I}=r S I-I \\
\dot{R}=I-b R
\end{array},\right.
$$

где параметр $b<<1$ характеризует скорость восстановления восприимчивости к возбудителю. Этот процесс, не позволяющий эпидемии окончательно затухнуть, меняет положение равновесия модели на $S^{*}=1 / r, I^{*}=b(r-1) / r(b+1)$. 
Если исключить лишнее третье уравнение, система принимает вид

$$
\left\{\begin{array}{l}
\dot{S}=-r S I+b(1-I-S) \\
\dot{I}=r S I-I
\end{array},\right.
$$

напоминающий модель «хищник-жертва» с восприимчивыми людьми в роли жертв, а инфицированными - в роли хищников. Однако жертвы здесь не размножаются, а пребывают извне, что делает колебания затухающими.

Линеаризация SIRS-модели вблизи точки $\left(S^{*}, I^{*}\right)$ приводит к квадратному уравнению на собственные числа $\lambda^{2}+\left(b+I^{*}\right) \lambda+I^{*}(1+b)=0$ с дискриминантом $D=I^{*}\left(I^{*}-4\right)+b^{2} \approx-4 I^{*}$. Таким образом, возникают колебания доли инфицированных, имеющие круговую частоту $\omega=\sqrt{I^{*}}$ и коэффициент затухания $\beta \approx b(1-1 / 2 r)$. Эти колебания можно рассматривать как волны эпидемии.

$\mathrm{SIR}(\mathrm{S})$-модель предполагает гомогенность популяции, хотя реальные популяции негомогенны в целом ряде аспектов. Далее рассматриваются два варианта гетерогенности условий заражения, которые можно условно охарактеризовать как социильную, связанную с индивидуальными различиями в интенсивности контактов, в которые вступают люди, и пространственную, связанную различиями общих условий взаимодействия, в которых люди пребывают.

Для первой модификации получены точные аналитические результаты, меняющие представления о природе репродуктивного числа, а для второй численный и приближенный анализ обнаруживает волны эпидемии, не связанные с нестойкостью иммунитета, действием сезонных факторов, мутациями возбудителя или эффектами запаздывания противоэпидемических мероприятий.

\section{СОЦИАЛЬНАЯ ГЕТЕРОГЕННОСТЬ}

Рассмотрим популяцию, которая состоит из людей типов $k=0,1,2, \ldots$, различающихся своей социальной активностью. Люди типа $k$ за единицу времени вступают в контакты с другими людьми в $m^{k}$ раз чаще людей типа 0 . Если при заражении человека его активность не меняется, то $S_{k}+I_{k}+R_{k}=a_{k}$, где $a_{k}-$ изначальные доли людей типа $k$, связанные нормировкой $\sum_{k} a_{k}=1$.

Динамика статусов для людей типа $k$ описывается уравнениями

$$
\left\{\begin{array}{l}
\dot{S}_{k}=-r m^{k} S_{k} I \\
\dot{I}_{k}=r m^{k} S_{k} I-I_{k}, \\
\dot{R}_{k}=I_{k}
\end{array},\right.
$$

где $I=\sum_{k} m^{k} I_{k}-$ сводная доля инфицированных людей. 
Аналогичным образом вводятся сводные доли восприимчивых и переболевших $S=\sum_{k} m^{k} S_{k}$ и $R=\sum_{k} m^{k} R_{k}$. Для сводных долей нормировка принимает вид $S+I+R=a$, где $a=\sum_{k} m^{k} a_{k}$, а их динамика описывается уравнениями

$$
\left\{\begin{array}{l}
\dot{S}=-r Z I \\
\dot{I}=r Z I-I, \\
\dot{R}=I
\end{array}\right.
$$

где $Z=\sum_{k} m^{2 k} S_{k}$.

Состояние $I=0$ (нет инфицированных людей ни одного типа) является положением равновесия модели, которое теряет устойчивость при $r Z>1$. Следовательно, в изначально полностью восприимчивой гетерогенной популяции эпидемия развивается при выполнении неравенства

$$
r \sum_{k} m^{2 k} a_{k}>1 \text {. }
$$

Это условие распространения эпидемии обобщает тривиальное условие $r>1$, имевшее место в случае гомогенной популяции.

Таким образом, реальное репродуктивное число определяется не столько трансмиссивностью возбудителя, сколько структурой социальной активности. Особо следует отметить, что в условие входит $m^{2}$, а не просто $m$, т.е. результат присутствия в популяции типов людей с большими $k$ - суперраспространителей - таков, как будто они только тем и заняты, что взаимодействуют друг с другом. Иначе говоря, болезнь передается в основном по сети суперраспространителей, от которых уже заражаются и люди с небольшими $k$.

Данный результат наглядно показывает, насколько сильно может меняться содержание привычных понятий при переходе от гомогенной к гетерогенной популяции. Особо показательным данный эффект оказывается, если популяция обладает маломировыми свойствами, типичными для социальных контактов.

В малом мире доля людей, имеющих $m$ связей, убывает как $m^{-(1+\alpha)}$. В обозначениях модели это распределение принимает вид $a_{k} \sim m^{-\alpha k}$, поскольку ширина бинов, соответствующих разным типам активности, нарастает экспоненциально, чем съедается единичка в показателе. Слагаемые в условии с точностью до нормировочного коэффициента равны $m^{(2-\alpha) k}$. Такая сумма сходится только при $\alpha>2$, в противном случае ее величина определяется верхним пределом суммирования, который потенциально неограничен. Однако для сетей социальных связей типичны показатели $1<\alpha \leq 2$, поэтому в малом мире распространение эпидемии практически неизбежно при сколько угодно малом $r$.

Завершая анализ социальной гетерогенности, выполним анализ устойчивости положения равновесия и с помощью способа, основанного на рассмотрении долей здоровых людей. Так, для доли восприимчивых людей типа $k$ можно 
записать уравнение $d S_{k} / S_{k}=-r m^{k} d R$. Его решение имеет вид $S_{k}=a_{k} x^{m^{k}}$, где введено обозначение $x=e^{-r R}$, т.е. доля восприимчивых людей каждого типа определяется сводной долей невосприимчивых. При этом сводная доля восприимчивых $S=\sum_{k} m^{k} a_{k} x^{m^{k}}$.

Подставив результат в уравнение на положение равновесия $1-S=R$, получаем формулу $r \sum_{k} m^{k} a_{k}\left(x^{m^{k}}-1\right)=\ln x$. Поделив обе ее стороны на $x-1$ и элиминировав тем самым тривиальное решение $x=1$, соответствующее затуханию эпидемии, приходим к уравнению на нетривиальные решения

$$
r \sum_{k} m^{k} a_{k} \frac{x^{m^{k}}-1}{x-1}=\frac{\ln x}{x-1} .
$$

Его левая сторона - возрастающая функция $x$, а правая - убывающая из бесконечности, т.е. решение может быть только одно. И чтобы оно существовало, максимум левой стороны, равный (с учетом того, что дробь там представляет собой сумму геометрической прогрессии с $m^{k}$ слагаемыми, каждое из которых не превосходит 1) $r \sum_{k} m^{2 k} a_{k}$, должен быть больше минимума правой, равного 1 , чем и воспроизводится условие развития эпидемии.

Пик эпидемии - максимум суммарной (не сводной) доли инфицированных - достигается при $\sum_{k} \dot{I}_{k}=(r S-1) I=0$, т.е. при $S=1 / r$. Поскольку все $S_{k}$ убывают, то пик может быть только один, иначе говоря, социальная гетерогенность не меняет качественного поведения модели.

\section{ПРОСТРАНСТВЕННАЯ ГЕТЕРОГЕННОСТЬ}

Социальные контакты являются парными, однако возможны и множественные взаимодействия, в ходе которых большое количество людей одновременно оказывается в условиях, благоприятствующих передаче инфекции (например, в замкнутом помещении). Если в такую ситуацию попадают $m$ человек, то пропорционально возрастают как вероятность того, что среди них есть инфицированные, так и число восприимчивых людей, которые будут заражены. При этом кинетика заражения меняет свой вид с $\dot{S}=-r S I$ на $\dot{S}=-r\left\langle m^{2}\right\rangle S I$, где осреднение производится по всем возможным множественным взаимодействиям. В них может вовлекаться различное число людей в населенных пунктах разного размера. Интенсивное использование общественного транспорта, посещение крупных торгово-развлекательных центров и возникновение прочих ситуаций, способствующих массовому скоплению людей, характерно, в первую очередь, для крупных городов.

Для простоты сведем всё разнообразие населенных пунктов к двум типам - столища и провинция. Между собой они связаны миграционными потоками, 
которые будем считать постоянными во времени, равномерными в пространстве и не зависящими от статуса людей. Кроме того, предположим, что инфекция изначально появляется в столице, а в провинцию переносится лишь за счет внутренней миграции. В этом случае доли людей разного статуса одинаковы во всех населенных пунктах провинции, так что их можно характеризовать единообразно. Уравнения модели для столицы (обозначения без черты) и для провинции (обозначения с чертой) принимают следующий вид:

$$
\left\{\begin{array} { l } 
{ \dot { S } = - r S I - p c ( S - \overline { S } ) } \\
{ \dot { I } = r S I - I - p c ( I - \overline { I } ) } \\
{ \dot { R } = I - p c ( R - \overline { R } ) }
\end{array} \text { и } \left\{\begin{array}{l}
\dot{\bar{S}}=-q r \bar{S} \bar{I}+c(S-\bar{S}) \\
\dot{\bar{I}}=q r \bar{S} \bar{I}-\bar{I}+c(I-\bar{I}) . \\
\dot{\bar{R}}=\bar{I}+c(R-\bar{R})
\end{array}\right.\right.
$$

Здесь базовые репродуктивные числа $r$ и $q r$ уже включают в себя результат множественных взаимодействий, параметр $q=\left\langle\bar{m}^{2}\right\rangle /\left\langle m^{2}\right\rangle<1$ соотносит интенсивности заражения в провинции и столице, параметр $p>>1$ равен отношению численностей населения провинции и столицы (они полагаются неизменными), а параметр $c<<1$ характеризует интенсивность миграционных потоков.

Благодаря нормировкам $S+I+R=1$ и $\bar{S}+\bar{I}+\bar{R}=1$ описание эпидемии требует по два уравнения из выписанных трех для столицы и провинции.

Хотя модель является четырехпараметрической, если провинциальное базовое репродуктивное число $q r<1$, то конкретная величина $q$ слабо влияет на развитие эпидемии, поскольку в провинции миграционные процессы доминируют над эпидемическими. Данное обстоятельство упрощает анализ модели.

Динамика решения при реалистичных значениях параметров показана на рис. 3. В столице на начальной стадии развития эпидемии доли восприимчивых и переболевших доходят практически до значений $S^{*}$ и $R^{*}$ при существенном падении доли инфицированных. Однако далее начинает сказываться миграционный обмен с провинцией, еще слабо затронутой эпидемией.

На фоне притока восприимчивых людей и оттока переболевших доля инфицированных в столице снова быстро возрастает, хотя уже не дотягивает до прежних значений. Поэтому и новые амплитудные значения $S$ и $R$, достигаемые при следующем падении $I$, оказываются дальше от $S^{*}$ и $R^{*}$, чем предыдущие. После некоторого числа таких колебаний доли восприимчивых и переболевших устремляются к асимптотическим значениям $S_{\infty}$ и $R_{\infty}$. Они отличны от $S^{*}$ и $R^{*}$, поскольку обуславливаются обращением в ноль производной $\dot{I}$ при пока еще положительных $I$ и $\bar{I}$. Полагая $\bar{I}<<I$ и $p c<<1$, получаем оценку $S_{\infty} \approx 1 / r$, убывающую с ростом $r$ лишь гиперболически, при экспоненциальном убывании $S^{*}$ для гомогенной модели. Иначе говоря, миграция восприимчивых людей из провинции приводит здесь к тому же качественному результату, что и утрата иммунитета переболевшими в SIRS-модели. 

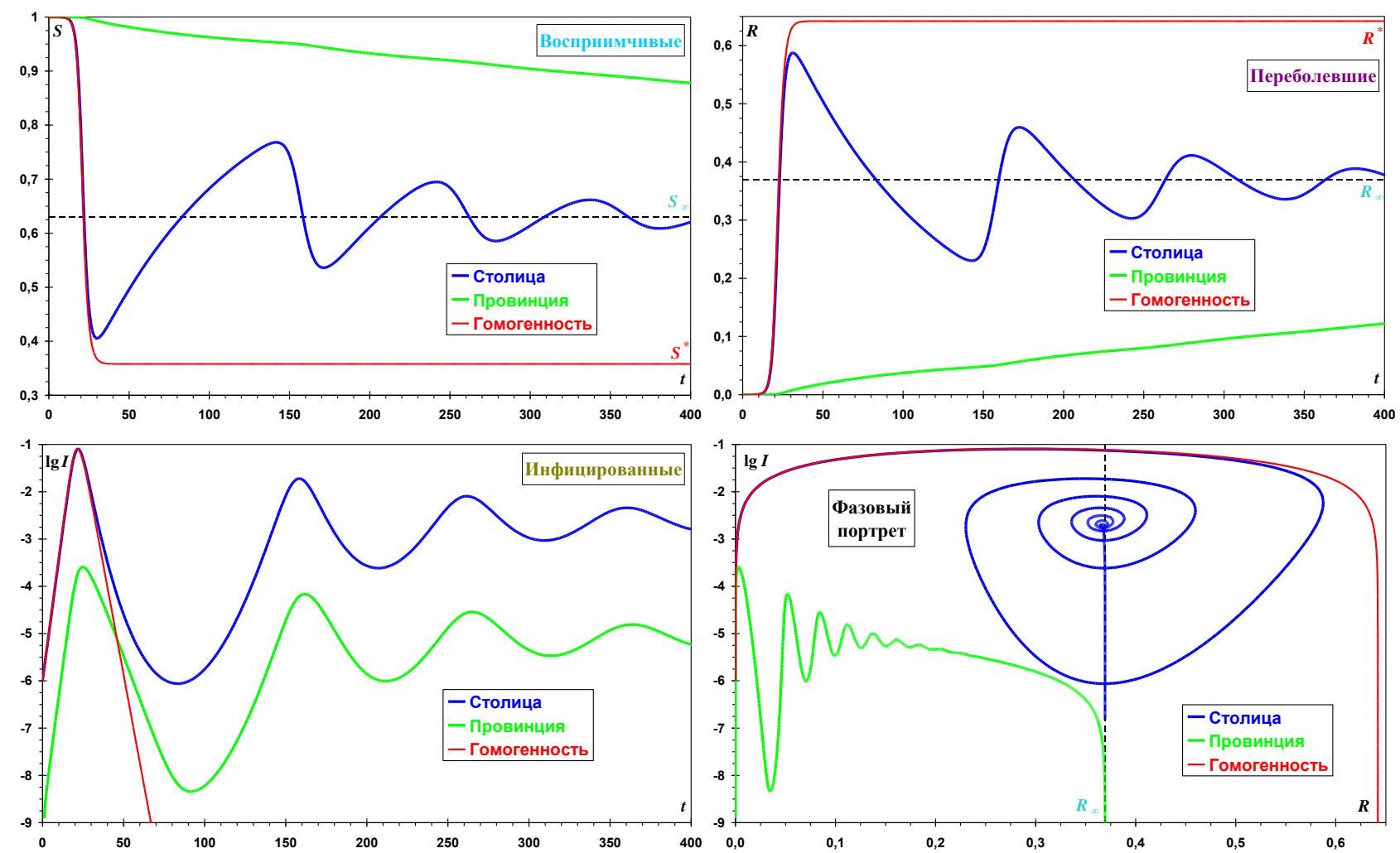

Рис. 3. Типовой случай возникновения волн эпидемии

Инфекционные фазы колебаний (убывающие для $S$ и возрастающие для $R$ ) являются резкими, тогда как миграционные фазы (возрастающие для $S$ и убывающие для $R$ ) - плавными. Провинциальная динамика инфицированных, определяемая преимущественно миграцией, почти синхронна со столичной.

После того, как колебания затухнут (при $t \sim 10^{3}$ ), а $\bar{S}$ и $\bar{R}$ приблизятся к $S_{\infty}$, и $R_{\infty}$, начинается этап экспоненциального убывания доли инфицированных (здесь на графиках от времени не поместился - см. рис. 4), выглядящий в координатах $(R, \lg I)$ как вертикальное падение.

Параметры: $r=1,6, q=0,5, p=10, c=10^{-3}$.

Провинциальные асимптотические значения, разумеется, совпадают со столичными, однако достигаются они лишь по мере миграционного перемешивания населения, т.е. очень медленно. К ним можно приблизиться и быстрее, если усилить миграцию. Однако, как можно видеть на рис. 4, в этом случае почти исчезают колебания, поскольку приток восприимчивых людей из провинции в столицу становится соизмерим по скорости с их заражением там.

После того, как доли здоровых в столице приближаются к асимптотическим значениям, динамика долей здоровых в провинции может быть удовлетворительно описана простыми уравнениями $\dot{\bar{S}}=c\left(S_{\infty}-\bar{S}\right)$ и $\dot{\bar{R}}=c\left(R_{\infty}-\bar{R}\right)$, откуда $\bar{S}-S_{\infty} \sim R_{\infty}-\bar{R} \sim e^{-c t}$. С другой стороны, из $S \approx S_{\infty}$ получается оценка $r S_{\infty} I \approx c p\left(\bar{S}-S_{\infty}\right)$, означающая очень медленное (по сравнению с гомогенной моделью) убывание доли инфицированных в столице $I \sim e^{-c t}$. А поскольку величина $I$ через миграцию ведет за собой и величину $\bar{I}$, их графики на рис. 4 оказываются параллельны в полулогарифмическом масштабе. 

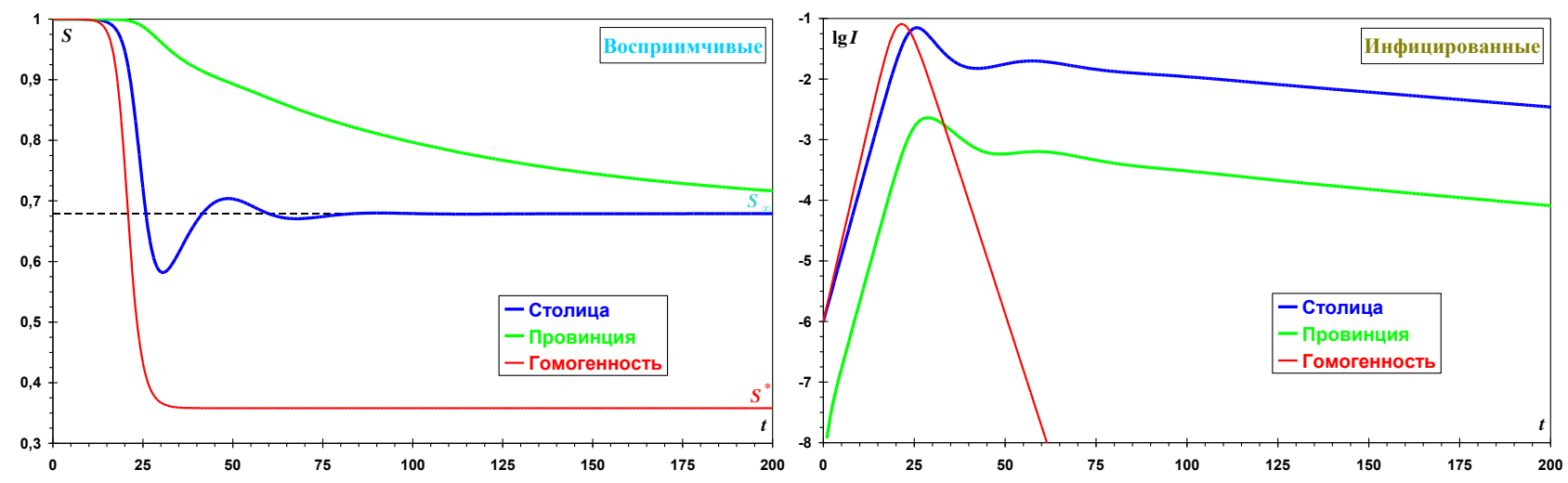

\section{Рис. 4. Подавление волн эпидемии при интенсивных миграционных потоках}

Из-за миграции доля восприимчивых не успевает сколь-нибудь близко подойти к $S^{*}$, в силу чего колебания почти сразу затухают. После этого начинается стадия экспоненциального убывания доли инфицированных, которая не поместилась в масштаб оси абсцисс на рис. 3.

Хотя затухание эпидемии и для гомогенной, и для гетерогенной моделей является экспоненциальным, его характерные времена для них оказываются очень разными, поскольку для первой оно обуславливается эпидемическими процессами, а для второй - миграционными.

Параметры: $r=1,6, q=0,5, p=10, c=10^{-2}$.

Если при рассмотрении волн эпидемии пренебречь миграцией инфицированных людей, а также изменением $\bar{S}$ на масштабе волны, то для столицы можно записать приближенные уравнения, похожие на SIRS-модель:

$$
\left\{\begin{array}{l}
\dot{S}=-r S I+p c(\bar{S}-S) \\
\dot{I}=r S I-I
\end{array} .\right.
$$

Линеаризуя их в окрестности точки $\left(S_{\infty}, I_{\infty}\right)$, где $I_{\infty}=p c\left(\bar{S}-S_{\infty}\right)$, получаем уравнение на собственные числа $\lambda^{2}+p c r \bar{S} \lambda+p c(r \bar{S}-1)=0$. С учетом $p c<<1$, его дискриминант $D \approx-4 p c(r \bar{S}-1)$. Таким образом, круговая частота малых колебаний $\omega=\sqrt{p c(r \bar{S}-1)}$, а коэффициент затухания $\gamma=p c r \bar{S} / 2 \approx p c r / 2$.

Однако колебания первых волн отнюдь не являются малыми, поскольку в их ходе доля инфицированных может изменяться на несколько порядков, т.е. период этих колебаний может существенно превосходить $T=2 \pi / \omega$. С другой стороны, с течением времени период колебаний тоже будет возрастать, но уже за счет падения $\omega$ при приближении $\bar{S}$ к $S_{\infty}$. Таким образом, в развитии эпидемии наступает момент, когда период колебаний минимален и волны идут наиболее часто. Именно для этого минимума следует ожидать наибольшей близости к полученным теоретическим результатам.

На рис. 5а минимальный интервал $T_{\min }$ между последовательными максимумами доли $I$, полученный при численном решении системы, сравнивается с нижней оценкой периода малых колебаний, соответствующей начальной ситуации с $\bar{S}=1$. Для весьма приближенных расчетов полученное совпадение можно считать удовлетворительным. 

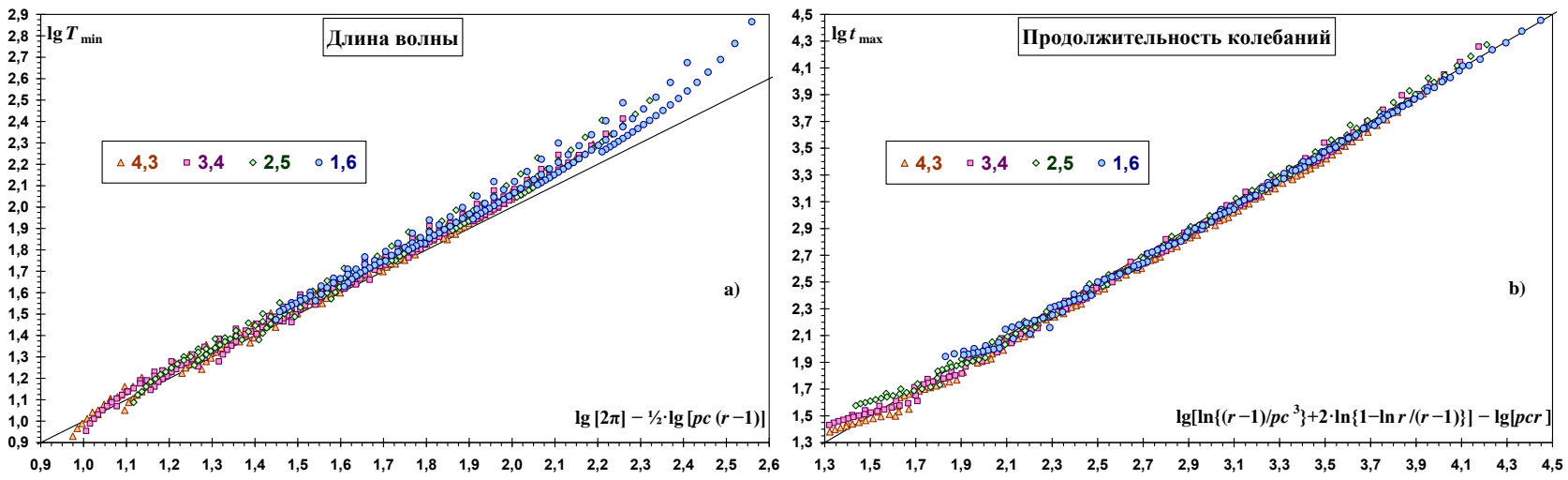

Рис. 5. Параметры волн эпидемии при различных значениях $r$

a) Для малых $p$ (особенно при небольших $r$ ) экспериментальная длина волн может в $1,5 \div 2,0$ раза превосходить ее теоретическую оценку. Величина $c$ сказывается на точности приближения слабее.

b) Отклонение времени, в течение которого продолжаются волны, от его теоретической оценки как в большую, так и в меньшую стороны не превышает 1,4 раза.

Параметры: $q=0,2, p=5 \div 25, c=2^{0 \div 7} \cdot 10^{-4}$.

При разумных значениях параметров период колебаний не опускается ниже десятков или даже сотен единиц времени, т.е. волны эпидемии в пространственно-гетерогенной системе сменяются медленно.

В рассматриваемой модели, в отличие от SIRS-модели, эпидемия проходит лишь через конечное число волн, после чего колебания прекращаются.

Оценим продолжительность колебательной стадии.

С учетом того, что $I_{\infty}<<I_{\max }$, динамику столичной доли инфицированных можно, полагая колебания гармоническими, приблизить в комплексном представлении формулой $I(t) \sim\left(I_{\infty}+I_{\max } e^{-\gamma t+i(\omega t+\varphi)}\right) \cdot e^{-c t}$. Положение локальных экстремумов этой величины определяется выражением

$$
\operatorname{Re}\left(-c I_{\infty}+(i \omega-\gamma-c) I_{\max } e^{-\gamma t+i(\omega t+\varphi)}\right) \approx-c I_{\infty}+\omega I_{\max } e^{-\gamma t}=0,
$$

где учтено $c<<\gamma<<\omega$. Экстремумы исчезают на временах, превышающих

$$
t_{\max }=\frac{1}{\gamma} \ln \frac{\omega I_{\max }}{c I_{\infty}} \approx \frac{1}{p c r} \ln \frac{(r-1)}{p c^{3}}\left(1-\frac{\ln r}{r-1}\right)^{2} .
$$

Как можно видеть из рис. 5b, эта оценка, хотя и построенная на тех же допущениях, что и оценка $T_{\min }$, лучше согласуется с результатами моделирования.

До настоящего момента модель рассматривалась только при параметрах с $q r<1$, не предполагающих самостоятельного развития эпидемии в провинции. В противном случае можно было бы ожидать тривиального поведения, схожего с гетерогенной моделью, при базовом репродуктивном числе, промежуточном между $q r$ и $r$. Однако в действительности, как позволяет убедиться рис. 6, волны всё-таки возникают и при $q r>1$. 

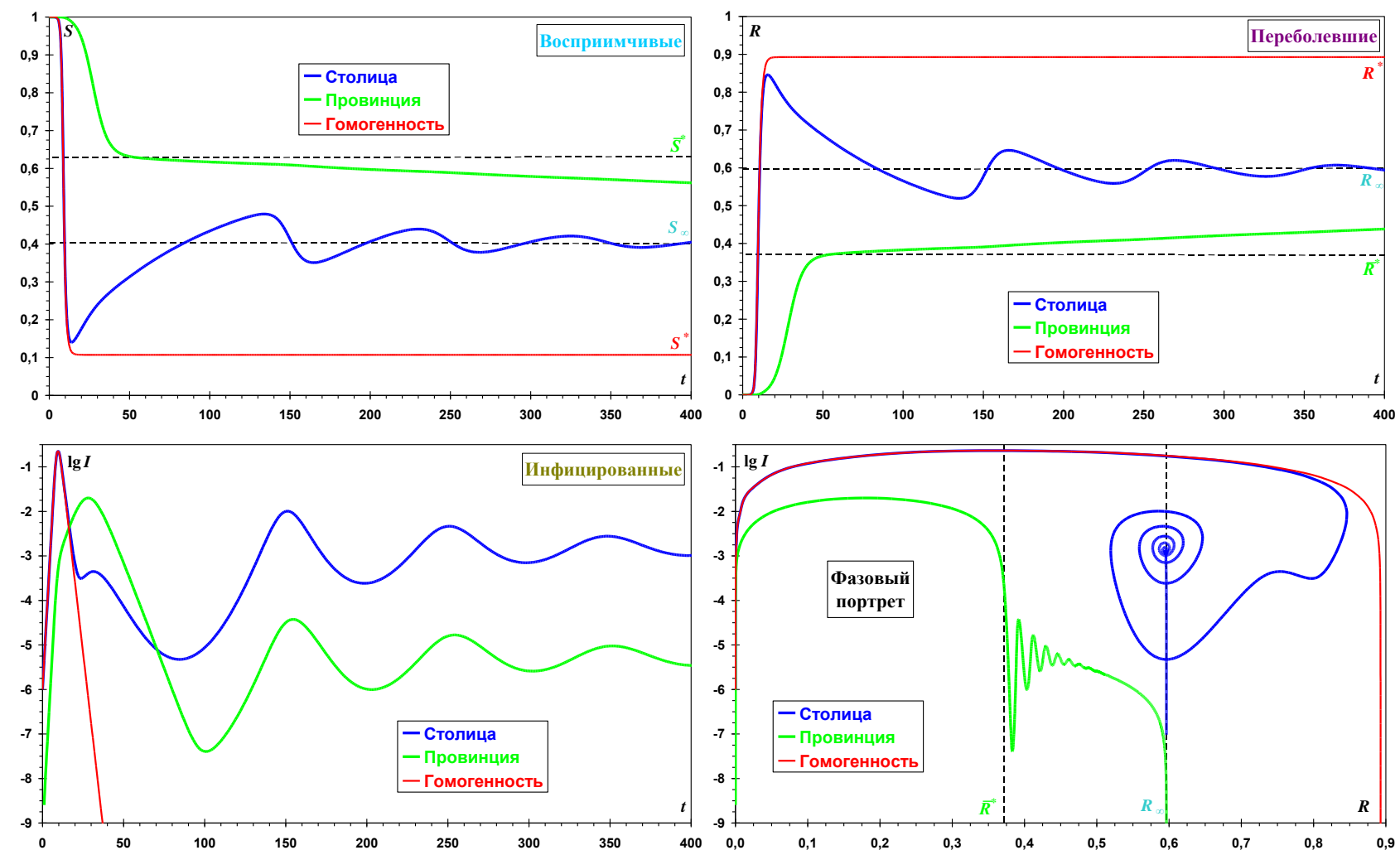

Рис. 6. Возникновения волн эпидемии при ее развитии и в столице и в провинции

На начальной стадии эпидемии доли восприимчивых людей в столице и в провинции быстро приближаются к $S^{*}$ и $\bar{S}^{*}$. Но поскольку в столице их остается больше, возникают волны.

Параметры: $r=2,5, q=0,5, p=10, c=10^{-3}$.

Такое поведение возможно, пока $\bar{S}^{*}>S_{\infty}$, т.е. если приобретение жителями провинции коллективного иммунитета не исчерпывает их возможности поставлять восприимчивых людей в столицу. Данное неравенство выполняется, пока $q<\ln r /(r-1)$. При этом провинциальное базовое репродуктивное число $q r$, вообще говоря, может быть сколь угодно большим при больших $r$.

\section{ВЫВОДЫ}

Различия в интенсивности парных контактов между людьми могут приводить к возникновению суперраспространителей, обеспечивающих развитие эпидемии при очень низкой, а в случае маломировых свойств сети контактов и при сколь угодно низкой трансмиссивности возбудителя.

Различия в интенсивности множественных контактов между людьми при наличии миграционных потоков могут приводить к изменению равновесных долей для различных статусов и порождать волны развития эпидемии. Примечательной особенностью колебательной динамики такой природы является ее ограниченность во времени. После прохождения через некоторое число волн эпидемия начинает затухать, хотя и намного медленнее, чем это происходит в гетерогенных условиях. 